\title{
BMJ Open Importance of personal and professional experience for hospital staff in person- centred dementia care: a cross-sectional interview study using freelisting in a UK hospital ward
}

Stephanie Petty, ${ }^{1,2}$ Tom Dening, ${ }^{\oplus 2}$ Amanda Griffiths, ${ }^{2}$ Donna Maria Coleston ${ }^{2}$

To cite: Petty S, Dening T, Griffiths A, et al. Importance of personal and professional experience for hospital staff in person-centred dementia care: a cross-sectional interview study using freelisting in a UK hospital ward. BMJ Open 2019;9:e025655. doi:10.1136/ bmjopen-2018-025655

- Prepublication history and additional material for this paper are available online. To view these files, please visit the journal online (http://dx.doi org/10.1136/bmjopen-2018025655).

Received 25 July 2018 Revised 23 November 2018 Accepted 30 January 2019

Check for updates

(C) Author(s) (or their employer(s)) 2019. Re-use permitted under CC BY-NC. No commercial re-use. See rights and permissions. Published by BMJ.

${ }^{1}$ Specialist Older Adults Psychology, The Retreat, York, UK

${ }^{2}$ Division of Psychiatry and Applied Psychology, School of Medicine, University of Nottingham, Nottingham, UK

Correspondence to Dr Stephanie Petty; spetty@theretreatyork.org.uk

\section{ABSTRACT}

Objective To detail how hospital staff with differing personal and professional caregiving experiences approach the care of patients with dementia, in order to make practical recommendations for practice.

Design Cross-sectional qualitative interviews.

Setting A UK hospital ward providing dementia care.

Participants A complete hospital ward staff team, constituting 47 hospital staff from 10 professions. Methods Hospital staff were asked to list their approaches to emotion-focused care in individual, ethnographic freelisting interviews. Cultural consensus analysis was used to detail variations in approaches to dementia care between staff subgroups.

Main outcome measures The most salient listed descriptions of care emphasised by staff members with personal experience of dementia caregiving when compared with staff members without such experience, and descriptions from staff newer to the profession compared with staff with more years of professional dementia caregiving experience.

Results Subgroups of hospital staff showed different patterns of responses both in how they noticed the emotional distress of patients with dementia, and in prioritised responses that they deemed to work. Hospital staff with professional experience of dementia caregiving and staff with fewer years of professional experience prioritised mutual communication and getting to know each patient.

Conclusions Subgroups of hospital staff with personal caregiving experiences and fewer years of professional care experience were more likely to describe personcentred care as their routine ways of working with patients with dementia. It is recommended that personal experience and the novice curiosity of hospital staff be considered as valuable resources that exist within multidisciplinary staff teams that could enhance staff training to improve the hospital care for patients with dementia.

\section{INTRODUCTION}

In the field of dementia care, there are initiatives to ensure that personal experience of
Strengths and limitations of this study

- We sought to discover the existing expertise within routine hospital care using the ethnographic freelisting method.

- The study builds on prior research recommendations to minimise future investments in interventions that rely on untested theoretical models of care.

- We sampled a representative hospital ward staff team that included different professions.

- The approach described by hospital staff does not necessarily equate to care delivered for all patients at all times.

- The findings require more robust testing and replication.

caregiving for somebody with dementia makes a substantial contribution to professional care. ${ }^{1}$ This is because a personal perspective can tailor care to address what matters most for the patient and can therefore improve health outcomes. ${ }^{2}$ These benefits are urgently needed in hospital care for patients with dementia, which has been addressed as an international priority ${ }^{34}$ and has been criticised for being task-orientated and falling short of person-centred care. ${ }^{56}$ By person-centred care, we mean that which meets the holistic needs of the patient as a person, who shares the same value and humanness as any other person. ${ }^{78}$ Finding ways to communicate with patients with dementia personally is particularly important because of the known difficulties with involving patients directly in their care. ${ }^{910}$ Prevalence estimates suggest that patients with dementia can occupy over a third of hospital beds in the $\mathrm{UK}^{11}$; there are serious implications of poor treatment compliance and wastage of care efforts when the patient's needs are not known. ${ }^{2}$ 
While personal experience of caregiving can be integrated into dementia care in hospitals by having family members present, ${ }^{12}$ this is limited by the physical and emotional demands on family members ${ }^{3}$ and the hospital priorities of managing risk and delivering medical care that fall within professional roles. ${ }^{5}$ Therefore, multidisciplinary hospital staff are required to deliver person-centred care. ${ }^{10}$

Quality hospital care has been evidenced but is variable ${ }^{13}$ and successful interventions to enhance person-centred care have been time-intensive and resource-intensive and with variable outcomes. ${ }^{14}$ An outstanding question remains as to how person-centred care can be achieved consistently by hospital staff. ${ }^{3515}$

We designed the current study in response to the call for research that explicitly seeks achievable solutions for routine practice and that recognises the existing skillset of hospital staff. ${ }^{35}$ This paper aims to detail the different, prioritised ways of working of hospital staff with varying personal and professional experiences of caregiving for patients with dementia. We seek to offer suggestions for enhancing care provision within the constraints of existing resources. Here, we focus specifically on the relationship shared with the patient at times of emotional distress as a component of person-centred care because of the challenge for both the patient and hospital staff at such times. ${ }^{16}$

\section{METHODS}

This study presents the analysis of intracultural variations in the approaches to dementia care across different subgroups within a hospital staff team. Specifically, we investigated whether staff with either personal experience of caregiving for a person with dementia or more years of professional experience than their peers approached care differently.

\section{Participants}

Forty-seven hospital staff members constituted a whole ward staff team over a 3-month period (October to December 2017), which included bank and temporary staff members and all shifts. Staff members were recruited from one ward for the assessment of older people within a teaching hospital in the UK. The ward was a member of Dementia Action Alliance, which connects 150 UK organisations through their commitment to improving dementia care; otherwise, the hospital had no dementia specialty such as consultation or liaison services and was not a dedicated dementia ward. The setting was chosen because of its similarity with hospital services for patients with dementia across Europe. ${ }^{17}$ All ward staff who interacted with patients within their working role were invited to participate, in an attempt to recognise whole system working. ${ }^{18}$ Study information was made available to all staff by the ward manager. Participants were informed of times when the researcher was available; all participants volunteered to take part and gave written informed consent prior to interview, after reading the study information. The hospital ward manager approved the study. The authors had no prior relationship with any participant. The lead author is a clinical psychologist, experienced in working with people across the age range with mental health diagnoses and their support networks.

\section{Patient and public involvement}

Staff from a second hospital in the UK were involved in the initial design of the content and format of the interview through discussion with the lead author to ensure that it was appropriate for use.

\section{Data collection}

Face-to-face, individual, freelisting interviews lasting $\sim 15$ min were conducted with all staff members in the ward team. Freelisting is an ethnographic method and provides the theoretical underpinning for the analysis. ${ }^{19}$ In the interview, staff were asked to keep in mind their working with patients with dementia and list as many items as they could to describe: (1) how they notice when a patient is emotionally distressed; (2) what they think causes patients to be emotionally distressed; (3) all the ways they respond when a patient is emotionally distressed; (4) of the ways to respond, all the things that seem to work. The type and severity of dementia was not specified. Interviews were audio recorded and transcribed in full. The transcripts were reviewed by all authors and list items were extracted through group discussion; $10 \%$ of the transcripts were reviewed independently by two authors.

\section{Data analysis}

Staff subgroups overview

Cultural consensus analysis ${ }^{19}$ was used to determine how different subgroups of staff described their approaches to care. Two sets of subgroups were created as follows. Personal caregiving experience: the list data of staff members who reported personal experience of dementia caregiving, such as having a family member or friend with the diagnosis $(n=18)$, was compared with the list data of staff without personal caregiving experience $(n=29)$; this formed datasets for two staff subgroups. Professional caregiving experience: the list data of staff members who had worked in a professional role with people with dementia for $>15$ years $(n=15)$ were compared with the list data of staff who had worked with people with dementia for $<5$ years $(n=18)$. This formed datasets for two further staff subgroups. The year boundaries chosen were a means of comparing staff with relatively more and fewer years of professional experience based on the demographic data in this study.

\section{Cultural consensus analysis}

ANTHROPAC analysis software was used in the following ways ${ }^{20}$ with close reference to example studies. ${ }^{21}{ }^{22}$ First, recode and consensus procedures were used to apply factor analytic statistical methods to determine whether each staff subgroup reached a shared domain description in response to each of the four interview questions. 
Consensus was shown by a single-factor solution, where the eigenvalues of the first factor and second factor formed a ratio of $>3: 1$. Second, each staff member's agreement with the consensus description of the subgroup was given by a knowledge score; this was each staff member's loading on the first factor, with a maximum loading of 1.0. This analysis showed whether different staff subgroups formed a consensus in their approach to care and how much each individual staff member agreed with the consensus.

\section{Salience of list items}

For each staff subgroup, the freelist procedure was used to calculate the listed items that were highest in salience for each of the four questions. ANTHROPAC applies Smith's salience index ${ }^{23}$ to measure how important an item is; an item with higher salience will have been mentioned more frequently and earlier in lists.

\section{Group comparisons}

Two main comparisons were made between (1) staff members with personal experience of dementia caregiving compared with those without and (2) staff members with more years of professional caregiving experience compared with those with fewer years of experience. For each comparison, the salience scores of items produced by one staff subgroup were subtracted from the salience scores of items produced by the second staff subgroup. This gave a list of difference scores ranging from positive values (items with higher salience for the first staff subgroup) to negative values (items with higher salience for the second staff subgroup). The list items at each end of the continuum show the emphasis of one group relative to the other. ${ }^{24}$ Qualitative differences in the items listed were then considered.

In addition, the mean number of items listed for each question was compared for staff with and without personal caregiving experience, and staff with more and fewer years of professional experience, using independent sample t-tests.

Finally, mean knowledge scores of each staff subgroup were compared with the mean knowledge score for the whole staff team for all four questions using paired sample t-tests; this shows the amount of agreement over the approach to care between members within a subgroup as compared with the full hospital ward.

The lead author performed all analysis.

\section{RESULTS}

\section{Participant overview}

All 47 members of the ward agreed to participate. The following professional roles were represented: healthcare assistant $(n=20)$, nurse or student nurse $(n=12)$, occupational therapist, physiotherapist or therapy assistant $(n=4)$, doctor $(n=3)$, manager or deputy manager $(n=3)$, domestic $(n=2)$, volunteer $(n=2)$ and ward clerk $(n=1)$. The majority of hospital staff were female $(70 \%)$ and White British (75\%), with an even spread of ages from across five age brackets, from ' 25 years or under' to ' 55 years or over'. The mean length of time working with people with dementia was 11 years (range 3 months to 37 years). Demographic differences between staff subgroups were both volunteers and three of four physiotherapists or therapy assistants had personal experience of caregiving; all other professions and genders were proportionately represented. The majority of staff without personal caregiver experience were aged $45-55$ years; the majority of staff with personal caregiver experience were aged 55 years or over. There were no differences of note in the representation of different professions or genders in the staff subgroups with more or fewer years of professional caregiving experience; however, all staff with more years of professional experience were aged 35 years or over and, collectively, were relatively older than staff with fewer years of professional experience, as might be expected.

\section{Comparing approaches to dementia care: personal experiences of caregiving \\ Shared domain descriptions}

Both staff subgroups produced a single, consensus domain description in response to each of the four interview questions. Both subgroups listed an equal number of items for all four questions; no comparison of mean number of items between staff subgroups for the four questions reached statistical significance. Therefore, neither group was more or less able to describe their approach to responding to the emotional needs of patients with dementia; however, meaningful differences were revealed in the amount of agreement between staff and in the different items listed.

\section{Staff agreement}

Knowledge scores showed that staff with personal experience of dementia caregiving showed less agreement with each other as a subgroup than they did with the whole staff team in their responses to all four questions, as shown by lower mean knowledge scores: ways to notice emotional distress $(\mathrm{t}(63)=4.21, \mathrm{p}<0.001)$, causes of emotional distress $(\mathrm{t}(62)=4.16, \mathrm{p}<0.001)$, responses to emotional distress $(\mathrm{t}(63)=2.41, \mathrm{p}=0.019)$ and responses that seem to work $(t(63)=2.96, p=0.004)$. In contrast, staff without personal experience of dementia caregiving did not differ significantly in their level of agreement with each other when compared with the whole staff team. Therefore, personal experience was influential when forming a consensus approach. This means that ward staff with personal experience of dementia caregiving showed more variety in how they noticed, understood and responded to patients with dementia.

\section{Comparison of list items}

Table 1A-D shows the list items with the greatest difference in salience between staff with personal caregiving experience as compared with staff without for all interview questions. 


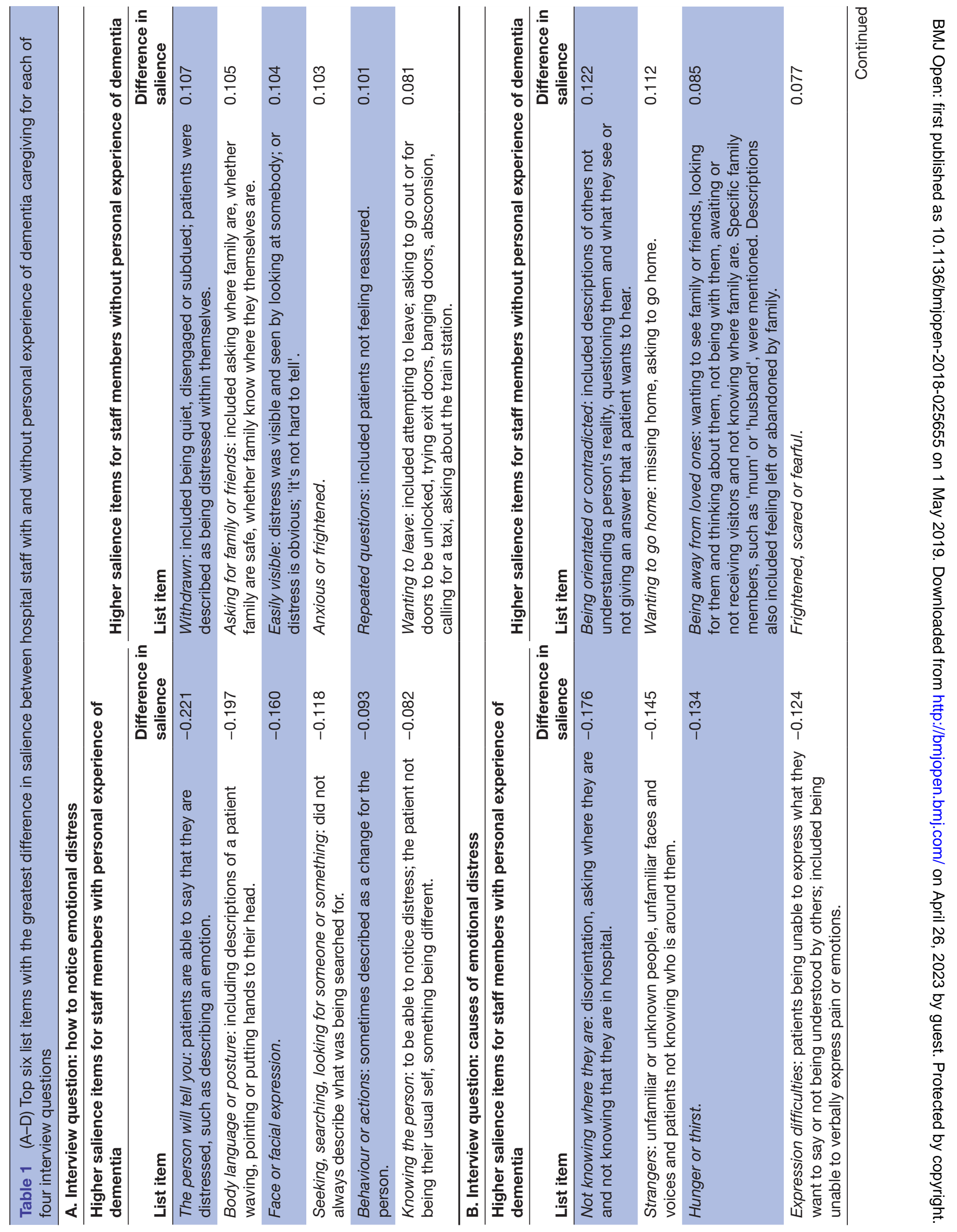




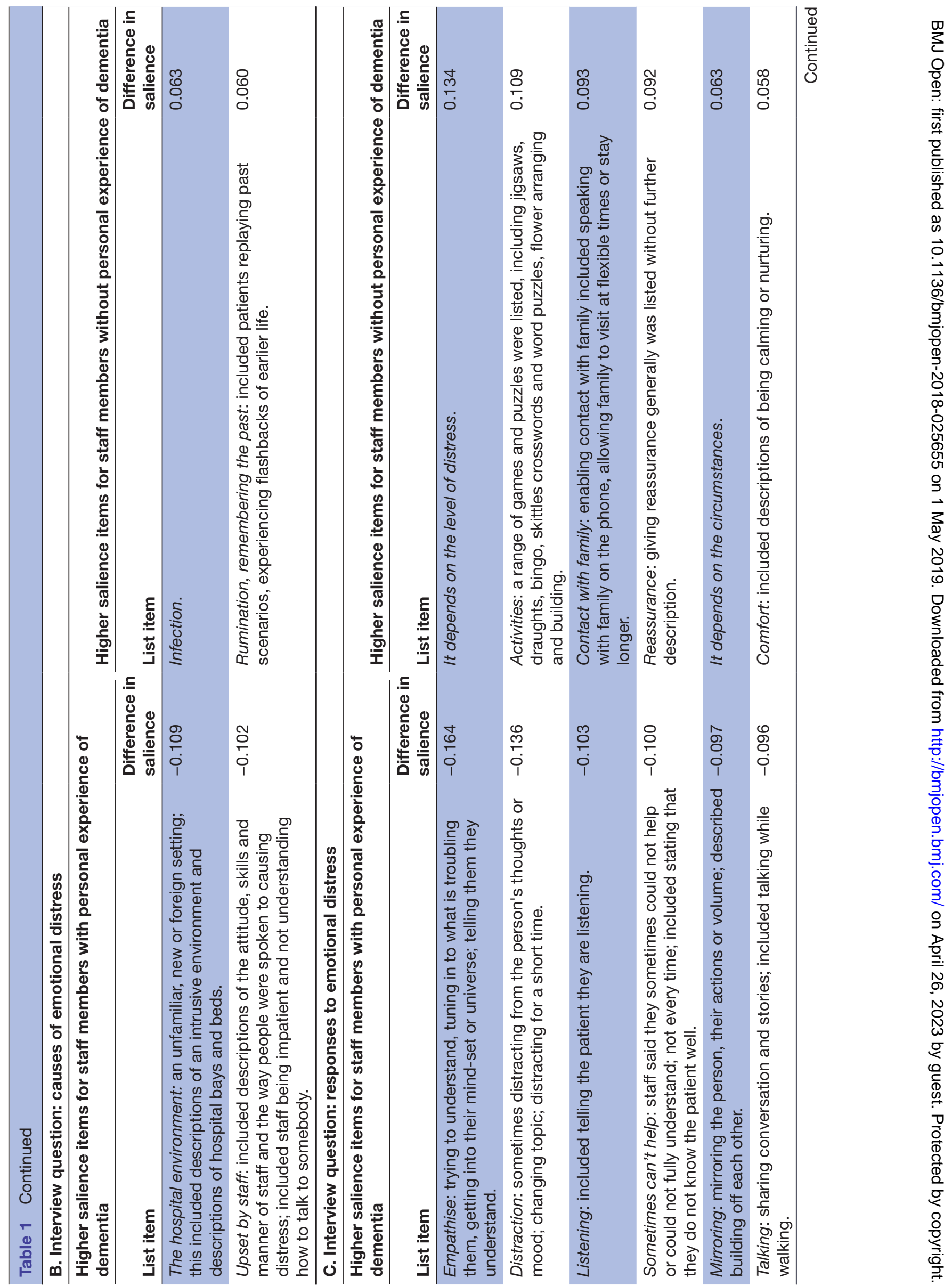




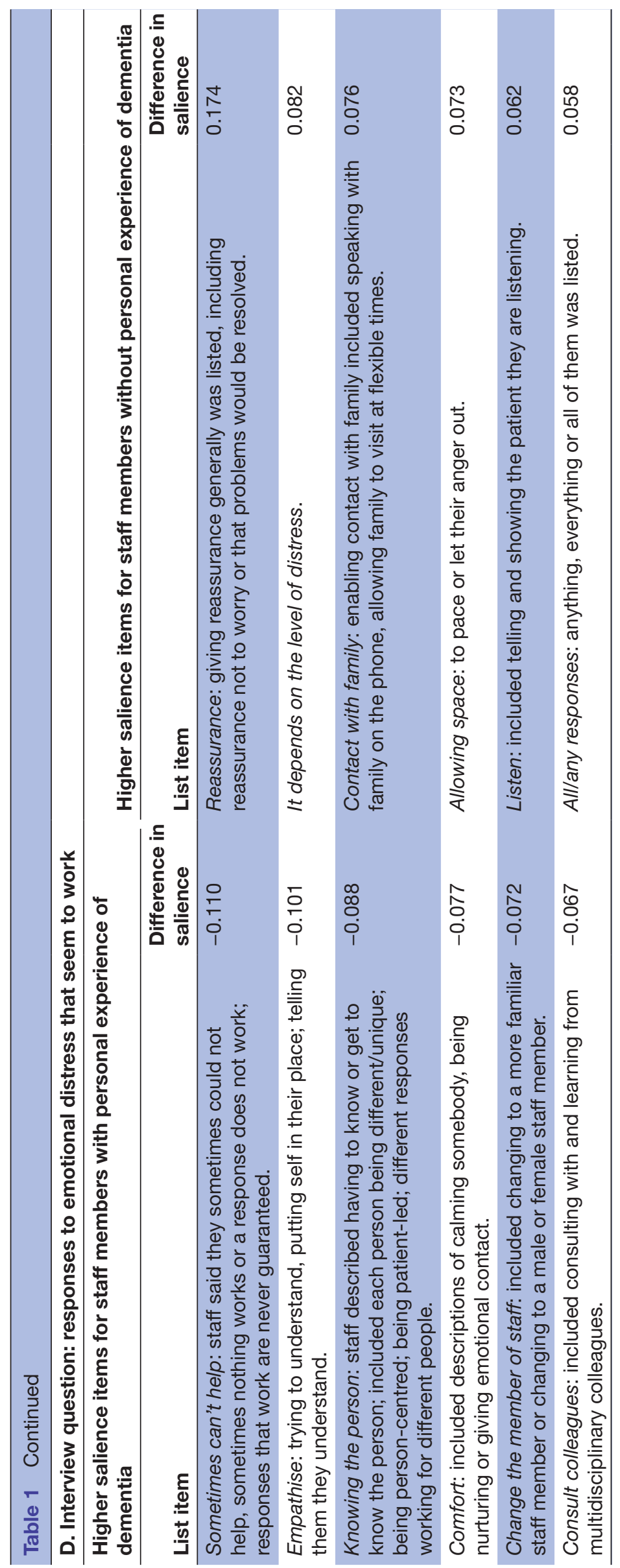


Staff with personal experience of dementia caregiving had a greater expectation that the patient would communicate their distress verbally or non-verbally through their facial expression or their body language. They emphasised that the staff member needs to know the patient as a person to be able to notice their emotional distress and their way of communicating. In their responses to emotional distress, this staff subgroup was more likely to report that they empathise, to talk, to listen and to mirror the patient. They were more likely to say that sometimes it is not possible to understand fully or respond helpfully. This suggests an approach to care, that is responsive to each individual and is personalised. This subgroup also placed stronger weighting on the hospital being a cause of distress, including being with strangers, being in an unfamiliar environment and feeling upset by the manner of hospital staff.

In contrast, ward staff without personal experience of dementia caregiving placed more emphasis on the role of a patient's family in maintaining their well-being: they recognised that a patient might ask for their family when distressed and recognised that causes of distress included being away from loved ones and wanting to go home. This subgroup also stated they would be more likely to respond to emotional distress by contacting a patient's family for a telephone call or visit. They showed more caution in how to respond to emotional distress: they were more likely to state that their response would depend on the level of distress or would depend on the circumstances and they were more likely to say that a wide range of responses to distress work at different times.

The approach described by all staff was nurturing, reassuring and comforting.

\section{Comparing approaches to dementia care: professional experiences of caregiving}

Online supplementary table S1A-D shows the list items with the greatest difference in salience between staff with more and fewer years of professional caregiving experience for all interview questions.

\section{Shared domain descriptions}

Both staff subgroups produced a single, shared domain description for each question; however, staff members with more years of professional experience listed significantly more items for ways to respond to emotional distress (mean 13.13, SD 4.94) than did staff with fewer years of professional experience (mean 8.33, SD 4.14) $(\mathrm{t}(31)=-3.04, \mathrm{p}=0.005)$, suggesting an accumulation of possible ways to respond to patients. They did not list significantly more responses that they deemed to work, as asked by the fourth question; this might suggest shared agreement between all staff of a limited number of effective responses.

\section{Comparison of list items}

Personalised care was more prevalent across responses to all questions for the staff subgroup with fewer years of professional experience. They were more likely to say that they noticed distress through easily visible cues, such as from a patient's face, or through their body language. They expected patients to voice their distress. They were more likely to say that they needed to know the patient as a person and that they would listen to the patient. In contrast, staff with more years of personal experience were more likely to use surmised terms when describing emotional distress; they listed agitation, aggression and anxiety, which might suggest a shorthand developed over time.

\section{DISCUSSION}

\section{Principal findings}

Hospital care for patients with dementia requires improvement and would benefit from clear recommendations that apply to routine practice. ${ }^{3-5}$ This study explored how existing resources of personal and professional caregiving experience could differentiate between the reported approaches of hospital staff when patients showed emotional distress. The results show that different staff subgroups emphasised varying features of person-centred care.

Staff members with personal experience of dementia caregiving prioritised knowing the person, achieving reciprocal communication and showing an understanding of the patient's perspective. The second staff subgroup comparison revealed complementary findings, whereby ward staff who were newer to a professional care role were more likely to notice each patient as a person and notice their individual communication. These findings make two notable contributions to the research literature. First, the approach described by these two staff subgroups, staff members with personal experience of dementia caregiving and staff members newer to professional caregiving, mirrors person-centred care $^{78}$ and exemplifies the most positive aspects of hospital care described in the research literature ${ }^{12}$ Second, these staff said that they communicated with the patient; hence, they involve patients in their care; such involvement is required as a fundamental standard of person-centred care and is particularly lacking for patients with dementia when in hospital. ${ }^{9}$

In previous research, ${ }^{25}$ hospital staff have expressed having more confidence in their working when they have personal experience of dementia caregiving. The current study adds to the literature by asking how staff with personal experience of dementia caregiving approach care when compared with their colleagues without such experience, using a cross-sectional design; the findings would support that personal experience of dementia caregiving contributes knowledge, confidence and a positive attitude to professional working as described elsewhere. ${ }^{25}{ }^{26}$ We also speculate as to whether career longevity promotes knowledge, but fosters a 'professional' approach to dementia care, whereby technical expertise and shorthand are valued for the purposes of 
documentation and risk management. ${ }^{6}$ This shorthand is consistent with expert thinking that has been refined over time, as compared with staff newer to the profession who make decisions more slowly and are influenced by more information, ${ }^{27}$ such as that relating to each individual patient. Traditional training in dementia care has prioritised medical care, ${ }^{5}$ which reflects the approach prioritised here by longer-standing staff. In this study, staff with more years of professional experience tended to describe behaviour as agitation or aggression and were more likely to list medical causes of emotional distress such as infection or delirium, which suggests an approach that overlooks the complexity of a person beyond diagnostic criteria. ${ }^{28}$ This interpretation does not dismiss the dedication of staff who sustain a career in working with patients with dementia, as was shown in the compassionate responses of all ward staff in this study. Instead, we aim to recognise the variations in approaches within a team.

We therefore recommend that the personal caregiving experiences and the novice curiosity of hospital staff delivering dementia care are considered to be two areas of expertise within staff teams. For example, the approaches described in this study could contribute valuable content to staff training interventions; training in the format of learning with colleagues and embedding learning in routine practice has been reported to be more effective in improving personalised dementia care than formal training interventions, which are not always suitable in their content and are not available to all staff. ${ }^{1425}$ The current findings support future investment in models of training, whereby colleagues who have differing expertise learn with and from each other. Flexible training formats, ${ }^{14}$ which emphasise collaborative learning and the sharing of existing expertise, are recommended.

The person-centred approach described could also be given merit by hospital management and clinical leaders. Hospital staff have expressed beliefs of having little authority or permission to influence routine patient care, ${ }^{5}$ though their contributions to service development and delivery can be particularly valuable in the delivery of person-centred dementia care. ${ }^{525}$ Hospital staff providing dementia care have asked that their existing knowledge and skills be recognised. ${ }^{5}$ This study shows the potential value of developing a hospital culture of staff learning together and sharing approaches that work.

\section{Strengths and weaknesses}

Strengths: primarily, we sought to discover the existing expertise within routine hospital care using the ethnographic freelisting method. This is important given the manifold barriers to hospital staff implementing best practice dementia care, such as having limited time. ${ }^{513} 1426$ We built on prior research recommendations to minimise future investments in interventions that rely on theoretical models of care only and have not been tested in a clinical setting; this is, to increase the real-world impact of the research. ${ }^{3}$ The findings offer some discussion as to how person-centred approaches could be enhanced, and how patients with dementia could be involved in their care. ${ }^{210}$ We sampled a representative hospital ward staff team that included different professions. Limitations: the approach described by ward staff does not necessarily equate to care delivered for all patients at all times. While we have detailed the knowledge of staff, we have not directly observed their behaviour. The generalisability of the findings is limited by the setting being a single hospital ward in the UK, and by the possible recall bias of participants when interviewed about their practice. The findings require more robust testing and replication. Future research would benefit from measuring staff knowledge, attitude and training in dementia care as possible confounding variables in the delivery of person-centred care, alongside measures of personal and professional caregiving experiences.

\section{Implications for clinicians and policymakers}

We intend the findings to influence staff training interventions, specifically, hospital staff have repeatedly expressed the value of collaborative learning with peers that these findings would encourage.$^{25}{ }^{29} \mathrm{We}$ also recommend that the person-centred approaches detailed here be given merit by management to enable change in the hospital culture. ${ }^{529}$ Possible benefits to sharing expertise among multiple professions include building a reliable skillset, that is, more resistant to staff turnover and is valuable when family members are not consistently available.

\section{Unanswered questions and future research}

These findings require follow-up in three ways: first, replication beyond a single UK hospital ward; second, further exploration of how patient, staff and the hospital system variables interact to complicate the delivery of person-centred care; third, evaluative studies of how personal experience and professional curiosity can be prioritised in practice.

The lead author affirms that this is an honest, accurate and transparent account of the study.

Acknowledgements The authors would like to thank all hospital staff for their contribution. They would also like to thank The Retreat York for their generous funding of S Petty's PhD studentship.

Contributors Study concept and design: SP, TD, AG and DMC. Acquisition of data: SP. Analysis and interpretation of data: SP, TD, AG and DMC. Drafting of the manuscript: SP. Critical revision of the manuscript: SP, TD, AG and DMC. As guarantor, SP accepts responsibility for the conduct of the study, had access to the data and controlled the decision to publish.

Funding This work was supported by The Retreat York as part of SP's PhD studentship.

Competing interests None declared.

Patient consent for publication Not required.

Ethics approval Ethical approval was granted by the Health Research Authority (ref 18/HRA/0221).

Provenance and peer review Not commissioned; externally peer reviewed.

Data sharing statement The authors will share relevant data on which the analysis, results and conclusions reported in the paper are based on reasonable request from BMJ Open. 
Open access This is an open access article distributed in accordance with the Creative Commons Attribution Non Commercial (CC BY-NC 4.0) license, which permits others to distribute, remix, adapt, build upon this work non-commercially, and license their derivative works on different terms, provided the original work is properly cited, appropriate credit is given, any changes made indicated, and the use is non-commercial. See: http://creativecommons.org/licenses/by-nc/4.0/.

\section{REFERENCES}

1. Worthington A, Rooney P, Hannan R. The triangle of care. Carers included: a guide to best practice in mental health care in England. 2nd edn. London, 2013.

2. Ocloo J, Matthews R. From tokenism to empowerment: progressing patient and public involvement in healthcare improvement. BMJ Qual Saf 2016;25:626-32.

3. Dewing J, Dijk S. What is the current state of care for older people with dementia in general hospitals? A literature review. Dementia 2016;15:1-19.

4. World Health Organization. Global action plan on the public health response to dementia 2017-2025. Geneva, Switzerland: World Health Organization, 2017.

5. Brooke J, Ojo O. Elements of a sustainable, competent, and empathetic workforce to support patients with dementia during an acute hospital stay: a comprehensive literature review. Int $\mathrm{J}$ Health Plann Manage 2018;33:e10-e25.

6. Digby R, Lee S, Williams A. The experience of people with dementia and nurses in hospital: an integrative review. J Clin Nurs 2017;26(910):1152-71.

7. Brooker DJ, Surr C. Dementia Care Mapping (DCM): initial validation of DCM 8 in UK field trials. Int J Geriatr Psychiatry 2006;21:1018-25

8. Kitwood T. The experience of dementia. Aging Ment Health 1997;1:13-22.

9. George J, Long S, Vincent C. How can we keep patients with dementia safe in our acute hospitals? A review of challenges and solutions. J R Soc Med 2013;106:355-61.

10. National Institute for Health and Care Excellence. Dementia. Assessment, management and support for people living with dementia and their carers. NICE Guideline [NG97]. London: National Institute for Health and Care Excellence, 2018.

11. Mukadam N, Sampson EL. A systematic review of the prevalence, associations and outcomes of dementia in older general hospital inpatients. Int Psychogeriatr 2011;23:344-55.

12. Scerri A, Innes A, Scerri C. Discovering what works well: exploring quality dementia care in hospital wards using an appreciative inquiry approach. J Clin Nurs 2015;24(13-14):1916-25.
13. Samra R, Griffiths A, Cox T, et al. Medical students' and doctors' attitudes towards older patients and their care in hospital settings: a conceptualisation. Age Ageing 2015;44:776-83.

14. Surr CA, Smith SJ, Crossland J, et al. Impact of a person-centred dementia care training programme on hospital staff attitudes, role efficacy and perceptions of caring for people with dementia: a repeated measures study. Int J Nurs Stud 2016;53:144-51.

15. Scerri A, Innes A, Scerri C. Dementia training programmes for staff working in general hospital settings - a systematic review of the literature. Aging Ment Health 2017;21:783-96.

16. Institute of Medicine. Crossing the quality chasm: a new health system for the 21st century. Washington DC: National Academy Press, 2001.

17. Hermann DM, Muck S, Nehen HG. Supporting dementia patients in hospital environments: health-related risks, needs and dedicated structures for patient care. Eur J Neurol 2015;22:239-45.

18. Innes A, Kelly F, Scerri C, et al. Living with dementia in hospital wards: a comparative study of staff perceptions of practice and observed patient experience. Int $J$ Older People Nurs 2016;11:94-106.

19. Weller SC, Romney AK. Systematic data collection. Newbury Park, California: Sage Publications, 1988.

20. Borgatti SP. ANTHROPAC 4.0. Natick, MA: Analytic Technologies, 1996.

21. Barg FK, Huss-Ashmore R, Wittink MN, et al. A mixed-methods approach to understanding loneliness and depression in older adults. J Gerontol B Psychol Sci Soc Sci 2006;61:S329-S339.

22. Schrauf RW, Sanchez J. Using freelisting to identify, assess, and characterize age differences in shared cultural domains. J Gerontol B Psychol Sci Soc Sci 2008;63:S385-S393.

23. Smith JJ. Using Anthropac 3.5 and a spreadsheet to compute a free list salience index: Cultural Anthropology Methods Newsletter, 1993;5:1-3

24. Schrauf RW, Sanchez J. The preponderance of negative emotion words in the emotion lexicon: a cross-generational and crosslinguistic study. J Multiling Multicult Dev 2004;25(2-3):266-84.

25. Griffiths A, Knight A, Harwood R, et al. Preparation to care for confused older patients in general hospitals: a study of UK health professionals. Age Ageing 2014;43:521-7.

26. Tropea J, LoGiudice D, Liew D, et al. Caring for people with dementia in hospital: findings from a survey to identify barriers and facilitators to implementing best practice dementia care. Int Psychogeriatr 2017;29:467-74.

27. Shanteau J. Competence in experts: the role of task characteristics. Organ Behav Hum Decis Process 1992;53:252-66.

28. Petty S, Dening T, Coleston DM, et al. Dementia: beyond disorders of mood. Aging Ment Health 2018:1-4.

29. Handley M, Bunn F, Goodman C. Dementia-friendly interventions to improve the care of people living with dementia admitted to hospitals: a realist review. BMJ Open 2017;7:e015257. 